\title{
Content-Aware Reverse Tone Mapping
}

\author{
Belen Masia* and Diego Gutierrez \\ ${ }^{1}$ Maria de Luna, 1. EINA. 50018-Zaragoza, Spain \\ ${ }^{*}$ Corresponding author
}

\begin{abstract}
We present an image analysis approach for reverse tone mapping of high dynamic range (HDR) content. The goal is to expand the dynamic range of existing low dynamic range content, to be properly shown on modern HDR displays. Most of the existing reverse tone mapping operators fail to offer a good solution in cases where the input images contain large saturated areas. In this paper we present an interactive, higher-level approach to reverse tone mapping. Inspired by the Zone System used in photography, it can also be used as an artistic tool where both the tonal balance and the mood of the final depiction can be adjusted by the user. Potential applications apart from visualization include algorithms for image or scene analysis.
\end{abstract}

Keywords-high dynamic range; reverse tone mapping; image analysis

\section{INTRODUCTION}

The increasing dynamic range of modern monitors enables them to display images with much greater contrast, similar to some real-world scenes. High dynamic range (HDR) content visualized on these monitors therefore recreates the real world more faithfully than low dynamic range (LDR) images viewed on conventional displays. However, there exists a great amount of legacy content which has been recorded and stored in low dynamic range formats, which now needs to be dealt with for a correct visualization on HDR displays.

Reverse tone mapping (rTM) refers to the process of expanding the range of an LDR input image or video to create an HDR depiction which matches real-world luminance values as faithfully as possible. Existing rTM operators work under the general assumption that highly saturated pixels need to be expanded much more than the rest. Even though these techniques can produce appealing results for a wide range of LDR content, there are some cases in which this approach may not be the best way to proceed, as shown recently by Masia and colleagues [1]. These cases include images -such as those shown in Figure I, which contain large saturated areas, either because of artistic purposes or due to a bad exposure. While the automatic detection of poorly exposed areas (as opposed to artistic choices) is still an open problem [2], we opt here for a user-in-the-loop approach.

We present two different techniques, one based in traditional photography, and another based on the detection of salient features; both allow the user to control dynamic range expansion based on her own preferences or intended goal. The techniques can also be used in combination with each other.
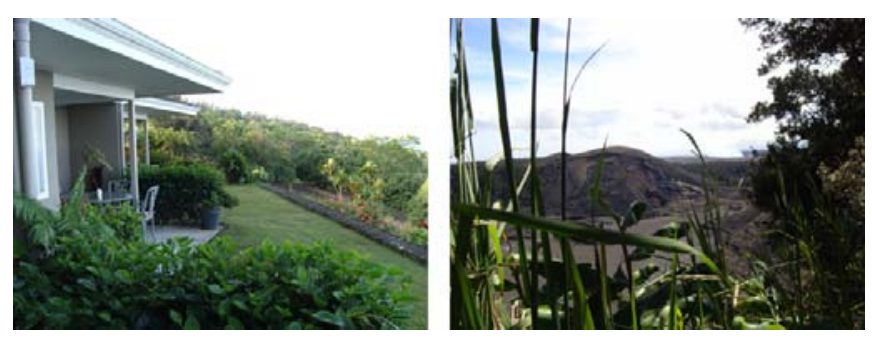

FIGURE I.

EXAMPLE IMAGES WITH LARGE SATURATED AREAS

\section{A PHOTOGRAPHIC APPROACH}

The so-called Zone System was introduced by Ansel Adams as a guide to produce good photographs with correct tonal values [3]. Following Adams' technique the luminance values in a scene can be divided into ten different luminance zones (0 through IX, see Figure II) [4]. Once the luminance range of the LDR input image is divided in zones in this way, our first proposed reverse tone mapping technique works by assigning different expansion functions to the different zones. Although in theory these functions could be as complex as desired, we choose to use linear functions for each zone, as they offer a good balance between simplicity and control over the expansion. Thus, the resulting rTM function is piece-wise linear. The darkest and the brightest zones ( 0 and IX, respectively) of the LDR image are mapped to the lowest and the highest luminance values of the HDR display. A second constraint is that the rTM function must be monotonically increasing, as otherwise gradient reversals may appear that spoil the final depiction. Adjusting the slopes of each of the zones may seem like an involved process; however, in the end it somehow resembles what photographers constantly do, as it translates to assigning ranges of the HDR image luminance to each zone of the LDR input image. Besides, the calculation of the resulting HDR image is almost immediate, thus allowing the user to try different curves before choosing thefinal one.

Figure III shows an example result; it depicts an expended HDR image obtained by using our proposed piece-wise linear curve, on which only three values were specified: Zone IV being assigned $10 \%$ of the HDR image luminance range, Zone VI is assigned $40 \%$ of that range, and Zone VII $50 \%$ of it, which translates into adjusting just three points of the LDR-HDR curve shown. We can also appreciate how this simple tuning of the rTM function yields a more appealing depiction than the linear scaling commonly used in most automatic dynamic range expansion techniques. 

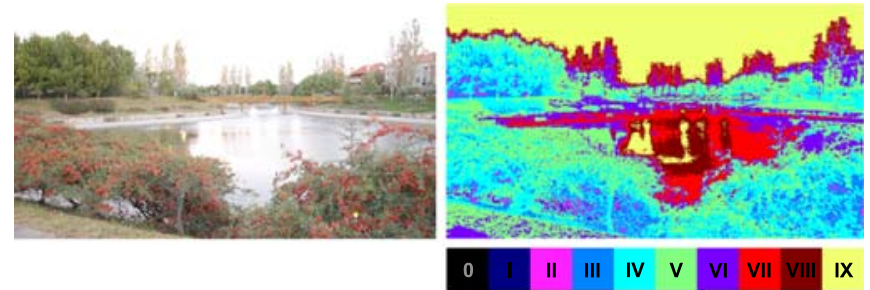

FIGURE II.

THE PHOTOGRAPHIC ZONE SYSTEM APPLIED

TO REVERSE TONE MAPPING. LEFT: INPUT IMAGE. RIGHT: LUMINANCE DECOMPOSITION WITH OUR PHOTOGRAPHIC APPROACH

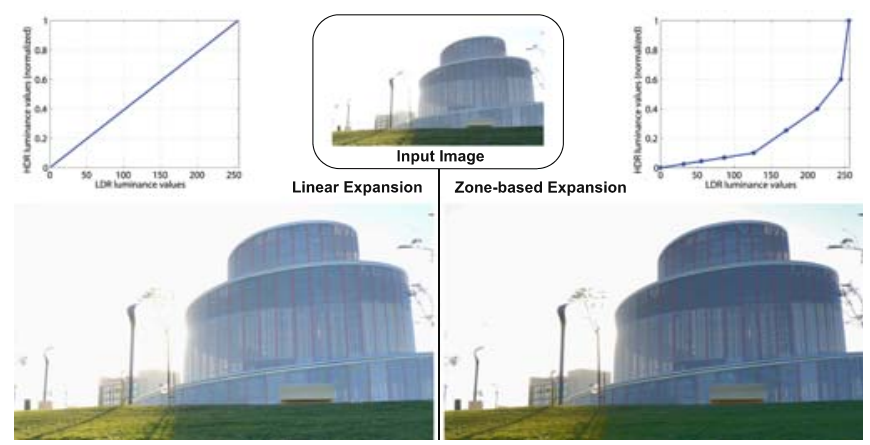

FIGURE III

AN EXAMPLE RESULT OF OUR PROPOSED REVERSE TONE MAPPING, BASED ON LUMINANCE DECOMPOSITION. TOP CENTER: ORIGINAL IMAGE. LEFT: HDR RESULT OBTAINED BY LINEAR EXPANSION. RIGHT: HDR RESULT USING OUR LUMINANCE-BASED EXPANSION, SHOWING A BETTER PRESERVATION OF DETAILS AND INCREASED CONTRAST. THE GRAPHS FOR BOTH EXPANSION TECHNIQUES ARE SHOWN ON TOP

\section{A SALIENCY-BASED APPROACH}

For our second approach, we propose to use a higher-level strategy, going beyond luminance analysis and taking into account the content of the scene. For this, we first detect the object of interest in order to use different reverse tone mapping functions for it and for the background. To separate the region of interest from the background we propose the use of a saliency detector. Saliency detection techniques pursue the objective of detecting those regions where the viewer's attention concentrates when looking at the image. Even though it is an active field where research continues to offer new and improved methods, a series of detectors exist which are able to offer convincing results in a wide variety of images. In general, saliency detection is performed by first, developing more or less complex models of the human visual system, and then using them in combination with image metrics. Following this, many existing methods obtain low level features on a first stage, and on a second stage they compute saliency based on these features. However, for many purposes it is necessary to perform a third stage in which object segmentation is applied to extract salient objects instead of just a map of salient locations. For our application, we rely on two different approaches that can applied indistinctively, summarized in the following subsections.

\section{A. Saliency Cuts}

This method (fully described in [5]) is essentially a combination of two techniques: the use of graph cuts for object segmentation, and a spectral approach to saliency detection [6]. Interactive graph cuts methods pose object segmentation as a minimal graph cuts problem. The nodes of the graph are formed by image pixels, and the two terminal nodes $\{\mathrm{s}, \mathrm{t}\}$ correspond to object and background, respectively. The marking of pixels as object or background by the user constitute the hard constraints on the problem, while soft constraints, which take into account boundary and region information, are also incorporated. The problem of finding minimal cuts in the graph is then solved via a max-flow algorithm. Figure IV (top) shows an example result of this saliency detection method.

The idea behind the Saliency Cuts framework is that even though interactive graph cuts can yield very accurate segmentations when proper priors are used, it usually requires a skillful user to select the appropriate regions to feed the algorithm. However, saliency regions can serve as seeds to the graph cuts segmentation process, thus obtaining an automatic and accurate separation between the salient object and the background. The limitations of the method lie in the fact that they can only detect a single object and in their assumption that the salient object is at the center of the image, while the sides are always assumed to belong to the background.

\section{B. Learning-Based Saliency}

In essence, this method formulates the problem as a Conditional Random Field (CRF) in which probabilities are inferred using a combination of salient features. Learning using a large training database is used to determine the optimal linear combination of the computed salient features. Given an input image, the goal is to obtain a binary saliency mask. We refer the reader to the work by Liu et al. for more details on this method [7]. The training stage is performed on a large database of about 21,000 images, where the salient object has been manually labeled.

Within this framework, the user can choose three different feature functions at different levels: multi-scale contrast at local level, center-surround histogram at regional level, and color spatial distribution at a global level. Our experiments confirm that the combination of all three yields an optimal result. Figure IV (bottom row) shows an example of these feature functions used and the final saliency mask obtained from them.

\section{Expanding Dynamic Range}

Once the division in object of interest and background has been performed, different expansion functions can be used for each. These expansion functions can be of any type. Given that we are focusing on an interactive approach where the user guides the reverse tone mapping process, we choose again to use piece-wise linear functions after a separation in luminance zones as explained before. Resulting HDR images obtained with our rTM framework and the corresponding saliencies and expansion functions are shown in the following section. 

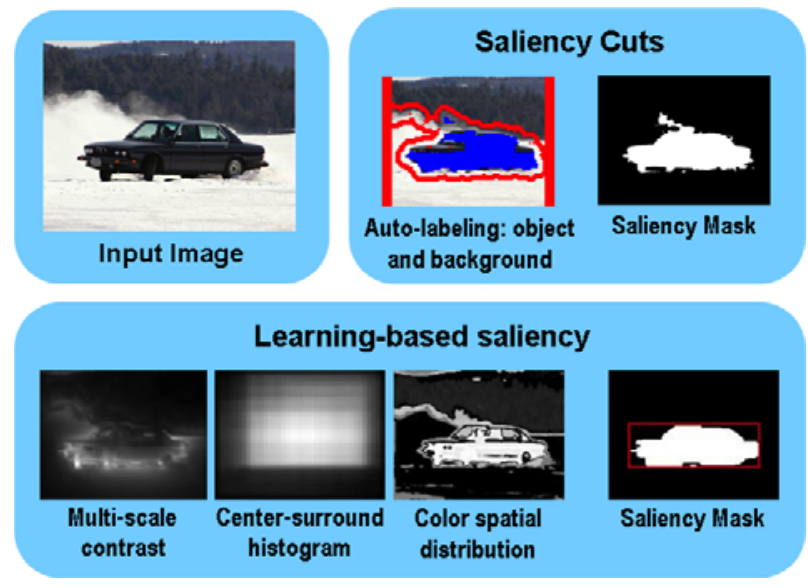

FIGURE IV. S SALIENCY DETECTION WITH OUR TWO SELECTED METHODS. TOP-LEFT: INPUT IMAGE. TOP-RIGHT: THE RESULT OF THE SALIENCY CUTS APPROACH. BOTTOM: THE RESULT OF THE LEARNING-BASED SALIENCY APPROACH
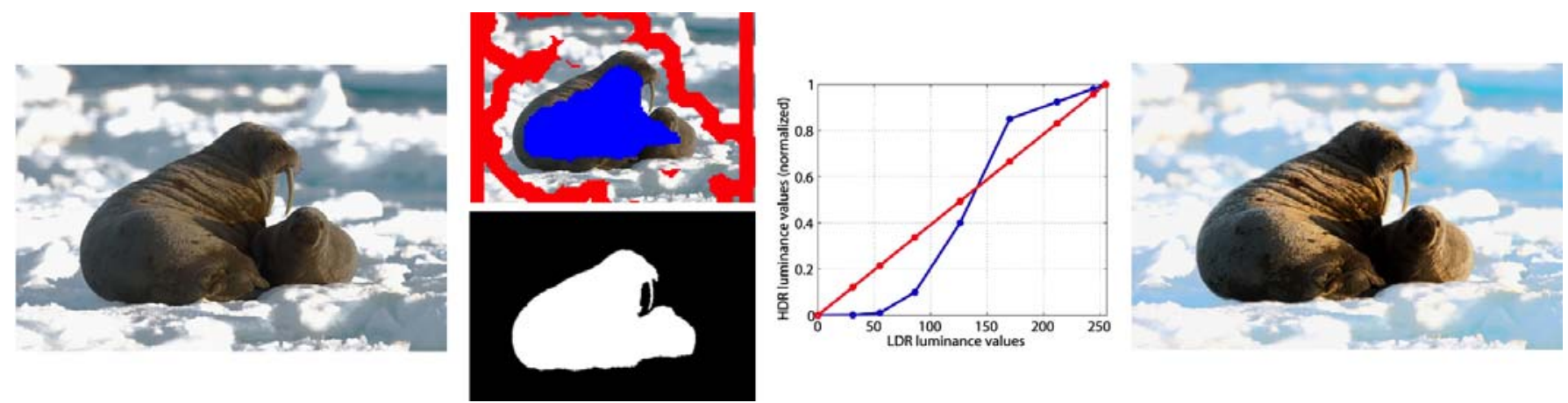

FIGURE V. COMPLETE PIPELINE USING OUR APPROACH. FROM LEFT TO RIGHT: INPUT LDR IMAGE; AUTO-LABELING OF THE SALIENT OBJECT (BLUE) AND THE BACKGROUND (RED), AND BINARY SALIENCY MASK; EXPANSION FUNCTIONS FOR THE SALIENT OBJECT (BLUE) AND THE BACKGROUND (RED); FINAL EXPANDED HDR IMAGE (TONE-MAPPED FOR VISUALIZATION PURPOSES)

In this first example, segmentation has been performed using Saliency Cuts (seeds used for the foreground and background are shown in blue and red, respectively). Even though both of the saliency detection methods presented produce segmentations accurate enough for our purposes given input images that are not excessively complex, for increasing complexity (either morphological or related to luminance values) manual segmentation may be necessary. The presence

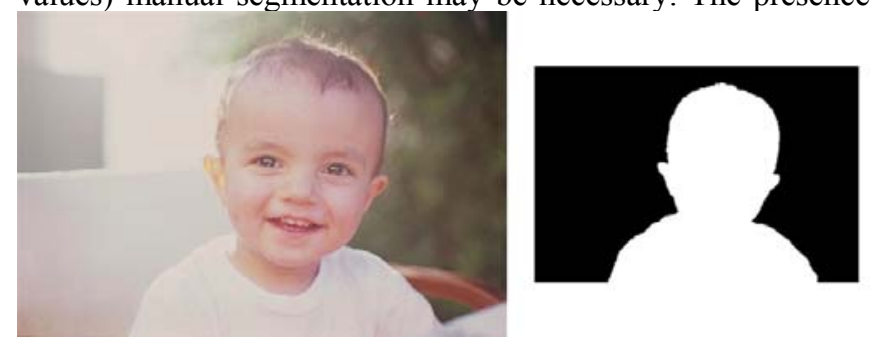

of more than two salient objects in the image would also require a manual segmentation, as the methods discussed cannot segment more than one object.

In the results presented in Figure VI, the object of interest (the kid's face) was segmented manually and, again, different zone-based piece-wise linear expansion functions were used for the salient object and for the background.

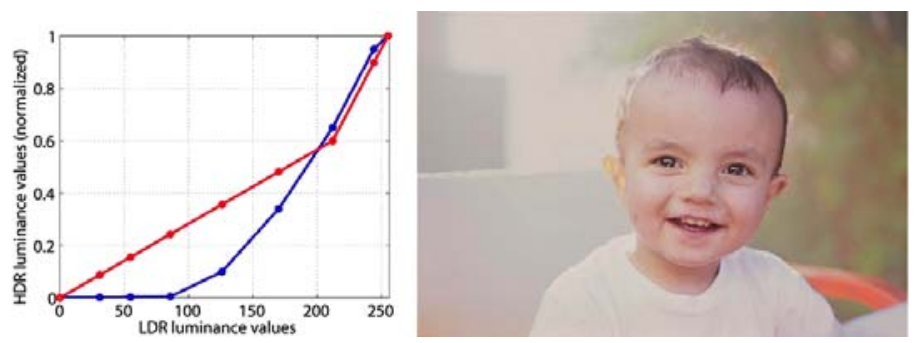

FIGURE VI.

REVERSE TONE MAPPING USING DIFFERENT ZONE-BASED EXPANSION FUNCTIONS FOR THE SALIENT OBJECT AND THE BACKGROUND. FROM LEFT TO RIGHT: INPUT LDR IMAGE; MANUALLY-OBTAINED SALIENCY MASK; EXPANSION FUNCTIONS FOR THE SALIENT OBJECT (BLUE) AND THE BACKGROUND (RED); FINAL EXPANDED HDR IMAGE (TONE-MAPPED FOR VISUALIZATION PURPOSES) 
The interactive nature of our approach implies that the functions for reverse tone mapping, which determine how the high dynamic range image will look, are adjusted and tuned with low dynamic range renditions of the images as real-time feedback. This is reasonable due to the fact that recent psychophysical experiments have demonstrated that the subjective quality of HDR images that have been generated from LDR images depends more on the presence of absence of spatial artifacts than on the exact luminance values, and thus a reasonably predictive evaluation of an HDR image can be done with an LDR depiction of it [1].

\section{CONCLUSIONS AND FUtURE WORK}

In this work we have presented an interactive approach to reverse tone mapping, which can be useful for a wide variety of images, and in particular those containing large saturated areas. The basis of our method is inspired by photographer Ansel Adams' well-known Zone System, which allows us to divide the luminance range of the image into zones. With the aid of this division in zones, and in an interactive process, a piecewise linear function to expand the LDR image can be provided by the user. Furthermore, our technique includes the possibility of using higher-level information as a guide for the expansion, segmenting the image in the object of interest and the background and using different expansion functions for each. This interactive approach offers a tool to expand the dynamic range of a scene with significant yet intuitive control over the final result. Besides, being able to freely adjust the luminance ranges of the zones makes it possible to obtain very different HDR depictions of the same input image, potentially providing an artistic tool for photographers and artists in general.

Regarding future work, adding a fitting step of the piecewise linear rTM functions proposed to smoother ones would be desirable. In the same sense, when dealing with content-aware rTM, taking care of the luminance transitions in the boundary between the objects of interest and the backgrounds would be necessary, either by somehow smoothing the binary mask or by placing constraints to the relationship between both -the object's and the background's- expansion functions.

Besides, thorough comparison between the proposed rTM technique and existing reverse tone mapping operators by means of psychophysical experiments would certainly be interesting for the field. Additionally, salient object detection is an open field of research, and our approach would definitely benefit from future advances in this field. Other lines of future research could involve the design of a contrast-based rTMO, working in the visual response space defined by the human visual system.

Another interesting line of future work would be to combine our content-aware approach with recent expansion methods based on a statistical analysis of the input image [8], or with a computational displays approach (see [9] for a recent review), taking into account the powerful combination of the joint design of optics, electronics, and algorithms.

\section{ACKNOWLEDGMENTS}

This research has been funded by the Spanish Ministry of Science and Technology (project LIGHTSLICE). Belen Masia would like to acknowledge the support of the Max Planck Center for Visual Computing and Communication. Diego Gutierrez was additionally funded through a Google Faculty Research Award.

\section{REFERENCES}

[1] Masia B., Agustin S., Fleming R. W., Sorkine O., Gutierrez D. Evaluation of reverse tone mapping through vary- ing exposure conditions. ACM Transactions on Graphics (SIGGRAPH Asia) 28, 5. 2009.

[2] Martin M., Fleming R.W., Sorkine O., Gutierrez D. Understanding exposure for reverse tone mapping. In CEIG 2008.

[3] Adams A. The Print. The Ansel Adams photography series. little, Brown and Company, 1983.

[4] Koren N. A simplified zone system (www.normankoren.com). Last retrieved October 2015.

[5] Fu Y., Cheng J., Li Z., Lu H. Saliency cuts: an automatic approach to object segmentation. In International Conference on Pattern Recognition, 2008.

[6] Hou X. D., Zhang L. Q. Saliency detection: a spectral residual approach. In Computer Vision and Pattern Recognition, 2007.

[7] Liu T., Sun J., Zheng N. N., Tang X., Shum H. Y. Learning to detect a salient object. In IEEE Computer Vision and Pattern Recognition, 2007.

[8] Masia B., Serrano A., Gutierrez D. Dynamic range expansion based on image statistics. Multimedia Tools and Applications, 2015.

[9] Masia B., Wetzstein G., Didyk P., Gutierrez D. A survey on computational displays: Pushing the boundaries of optics, computation and perception. Computers \& Graphics 37, 8. 2013. 